\title{
Infrared dielectric properties of low-stress silicon nitride
}

\author{
Giuseppe Cataldo, ${ }^{1,2, *}$ James A. Beall, ${ }^{3}$ Hsiao-Mei Cho, ${ }^{3}$ Brendan McAndrew, ${ }^{1}$ \\ Michael D. Niemack, ${ }^{3}$ and Edward J. Wollack ${ }^{1}$ \\ ${ }^{1}$ NASA Goddard Space Flight Center, 8800 Greenbelt Road, Greenbelt, Maryland 20771, USA \\ ${ }^{2}$ Universities Space Research Association, 10211 Wincopin Circle, Columbia, Maryland 21044, USA \\ ${ }^{3}$ National Institute of Standards and Technology, 325 Broadway, Boulder, Colorado 80305, USA \\ *Corresponding author: Giuseppe.Cataldo@nasa.gov
}

Received April 26, 2012; revised September 7, 2012; accepted September 10, 2012; posted September 10, 2012 (Doc. ID 167420); published October 4, 2012

\begin{abstract}
Silicon nitride thin films play an important role in the realization of sensors, filters, and high-performance circuits. Estimates of the dielectric function in the far- and mid-IR regime are derived from the observed transmittance spectra for a commonly employed low-stress silicon nitride formulation. The experimental, modeling, and numerical methods used to extract the dielectric parameters with an accuracy of approximately $4 \%$ are presented. (C) 2012 Optical Society of America

OCIS codes: $\quad 310.3840,310.6188,310.6860$.
\end{abstract}

The physical properties of silicon nitride thin films, namely low tensile stress, low thermal/electrical conductance, and its overall compatibility with other common materials, have facilitated its use in the microfabrication of structures requiring mechanical support, thermal isolation, and low-loss microwave signal propagation (e.g., [1-4]). Silicon nitride films are amorphous, highly absorbing in the mid-IR [ㄷ], and their general properties are functions of composition [6,, 7$]$. Here the optical properties are studied in detail for a membrane with parameters commonly employed in microfabrication.

The silicon nitride optical test films were prepared by a low-pressure chemical-vapor-deposition (LP-CVD) process optimized for low tensile stress and refractive index []. The $5: 1 \mathrm{SiH}_{2} \mathrm{Cl}_{2} / \mathrm{NH}_{3}$ gas ratio employed results in a tensile stress $<100 \mathrm{MPa}$ and optical index greater than $\sim 2$ [9]. The test structure is shown schematically in Fig. 1 (inset). Double-side-polished silicon $(75 \mathrm{~mm}$ diameter, $500 \mu \mathrm{m}$ thick) wafers [10] were used as a mechanically robust handling structure for the $\mathrm{SiN}_{x}$ membranes. A $150 \mathrm{~nm}$ thermal oxide was grown on the silicon wafers by wet oxidation at $950^{\circ} \mathrm{C}$ for $31 \mathrm{~min}$. This layer was subsequently used as an etch stop to protect the nitride during definition of the silicon handling wafer geometry. A low-stress $\mathrm{SiN}_{x}$ layer was then deposited by LP-CVD [e.g., deposition parameters for $2 \mu \mathrm{m}$ film are $835^{\circ} \mathrm{C}$ for $9.7 \mathrm{~h}$ with pressure $33 \mathrm{~Pa}$ and $12 \mathrm{sccm} \mathrm{NH3}, 59 \mathrm{sccm} \mathrm{SiH}_{2} \mathrm{Cl}_{2}$ (SCCM denotes cubic centimeters per minute at standard temperature and pressure)]. The wafers were then patterned with a resist mask and $\mathrm{SiN}_{x} / \mathrm{SiO}_{2}$ windows formed by deep reactive ion etching, which removed all the silicon under the window area. The residual thermal oxide was removed with hydrogen fluoride vapor etch, leaving a set of uniform $\mathrm{SiN}_{x}$ membranes each with a $10 \mathrm{~mm}$ diameter aperture individually suspended from the silicon handling frame.

The optical tests were performed on $\mathrm{SiN}_{x}$ samples having membrane thicknesses of 0.5 and $2.3 \mu \mathrm{m}$ with a uncertainty of 3\%. Fabry-Perot resonators were made by stacking multiple samples with silicon standoff frames between adjacent samples to explore the longwavelength response of the material in greater detail. The silicon standoffs allowed a vent path for evacuation of air between the nitride membranes. All optical measurements were performed in vacuum with a residual pressure less than $100 \mathrm{~Pa}$.

The samples were characterized with a Bruker 125 highresolution Fourier transform spectrometer (FTS) and were measured in transmission at the focal plane of an $f / 6$ beam. A number of different sources, beam splitters, and detector configurations were used in combination to provide measurements over the reported spectral range. The single-layer $\mathrm{SiN}_{x}$ sample transmission was measured over an extended range from 15 to $10,000 \mathrm{~cm}^{-1}$. The mercury lamp and a multilayer Mylar beam splitter were used to access frequencies below $600 \mathrm{~cm}^{-1}$. Additional mid-IR spectral data up to $2400 \mathrm{~cm}^{-1}$ were acquired using a ceramic glow bar source, Ge-coated $\mathrm{KBr}$ beam splitter, and room-temperature deuterated tri-glycine sulfate detector. The remaining near-IR data up to $10,000 \mathrm{~cm}^{-1}$ were taken with a $W$ filament source, $\mathrm{Si}$ on $\mathrm{CaF}_{2}$ beam splitter, and a liquid-nitrogen-cooled InSb detector (Fig. 1). Far-IR data between 15 and $95 \mathrm{~cm}^{-1}$ were taken using a mercury arc

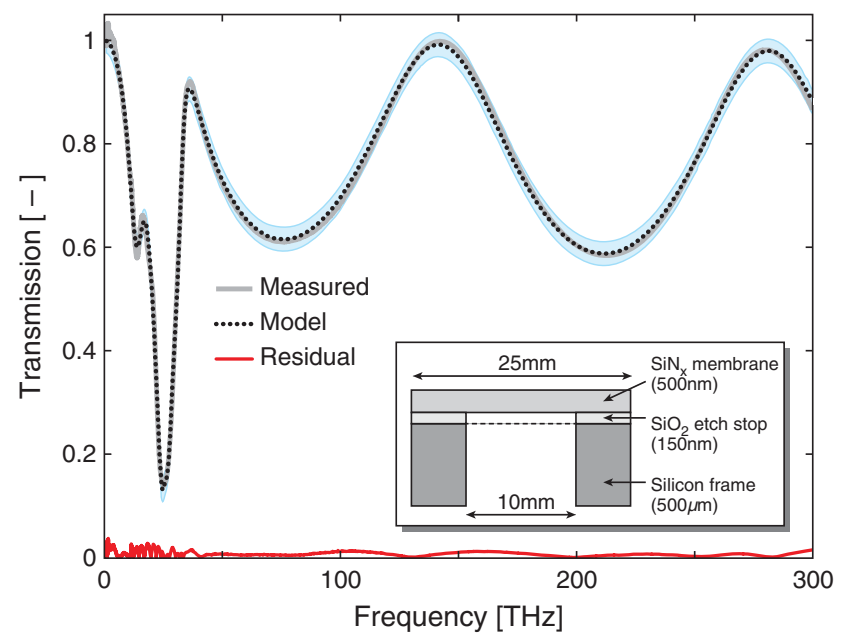

Fig. 1. (Color online) Room-temperature transmission of a silicon nitride sample $0.5 \mu \mathrm{m}$ thick: measured (grey), model (black dotted), and residual (red). The shaded band's width delimits the estimated $3 \sigma$ measurement uncertainty. A $30 \mathrm{GHz}$ $\left(1 \mathrm{~cm}^{-1}\right)$ resolution is employed for the measurement. The insert depicts the geometry of the $\mathrm{SiN}_{x}$ membrane and micromachined silicon frame. 


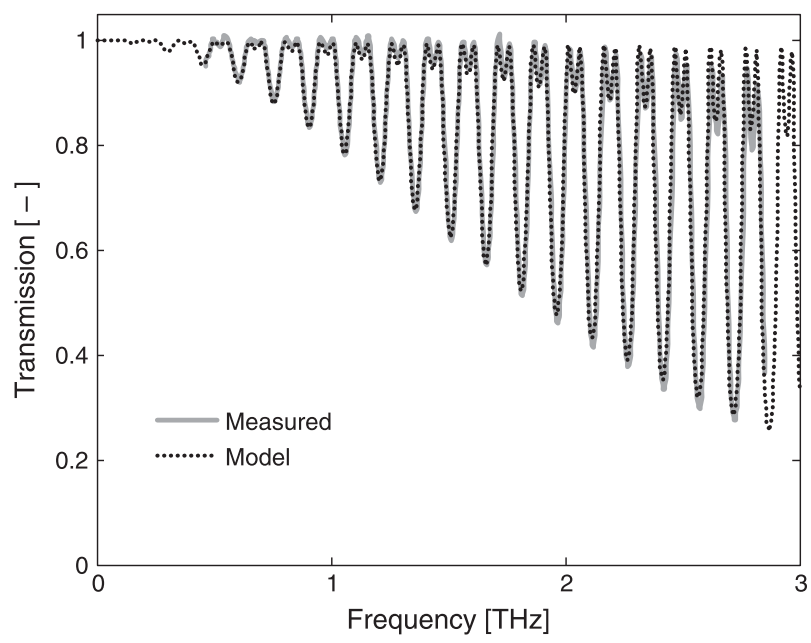

Fig. 2. Measured (solid grey) and model (black dotted) transmission for a three-layer stack of silicon nitride samples $2.3 \mu \mathrm{m}$ in thickness with $998 \mu \mathrm{m}$ intermembrane delays that complements the data shown in Fig. 1. The sample response in the far-IR was acquired with a resolution of $3 \mathrm{GHz}\left(0.1 \mathrm{~cm}^{-1}\right)$.

lamp source and a liquid-helium-cooled $4.2 \mathrm{~K}$ bolometer. Mylar beam splitters of 50, 75, and $125 \mu \mathrm{m}$ thicknesses and a multilayer Mylar beam splitter were used during separate scans (Fig. 2). The resultant transmission data were merged into a single spectra using a signal-to-noise weighting for subsequent parameter extraction.

The dielectric response is represented as a function of frequency, $\omega$, by the classical Maxwell-Helmholtz-Drude dispersion model [11],

$$
\hat{\varepsilon}_{r}(\omega)=\hat{\varepsilon}_{\infty}+\sum_{j=1}^{M} \frac{\Delta \hat{\varepsilon}_{j} \cdot \omega_{T_{j}}^{2}}{\omega_{T_{j}}^{2}-\omega^{2}-i \omega \Gamma_{j}^{\prime}(\omega)},
$$

where $M$ is the number of oscillators and $\hat{\varepsilon}_{r}=\varepsilon_{r}^{\prime}+i \varepsilon_{r}^{\prime \prime}$ is a complex function of $(5 M+2)$ degrees of freedom, which are as follows: the contribution to the relative permittivity $\hat{\varepsilon}_{\infty}=\hat{\varepsilon}_{M+1}$ of higher lying transitions, the difference in relative complex dielectric constant between adjacent oscillators $\Delta \hat{\varepsilon}_{j}=\hat{\varepsilon}_{j}-\hat{\varepsilon}_{j+1}$, which serves as a measure of the oscillator strength, the oscillator resonance frequency $\omega_{T_{j}}$, and the effective Lorentzian damping coefficient $\Gamma_{j}^{, j}$, for $j=1, \ldots, M$. The following functional form is used to specify the damping:

$$
\Gamma_{j}^{\prime}(\omega)=\Gamma_{j} \exp \left[-\alpha_{j}\left(\frac{\omega_{T_{j}}^{2}-\omega^{2}}{\omega \Gamma_{j}}\right)^{2}\right],
$$

where $\alpha_{j}$ allows interpolation between Lorentzian $\left(\alpha_{j}=0\right)$ and Gaussian wings $\left(\alpha_{j}>0\right)$ similar to the approach in [12]. The form indicated above enables a more accurate representation of relatively strong oscillator features.

The impedance contrast between free space and the thin-film sample forms a Fabry-Perot resonator. The observed transmission can be modeled [13] as a function of the dielectric response [Eq. (1)], thickness, and wavenumber. The dielectric parameters were solved by means of a nonlinear least-squares fit of the transmission equation to the laboratory FTS data. Specifically, a sequential quadratic programming method with computation of the Jacobian and Hessian matrices [14,15] was implemented. The merit function, $\chi^{2}$, was used in a constrained minimization over frequency as follows:

$$
\min _{\mathrm{DOF}} \chi^{2}=\min _{\mathrm{DOF}} \sum_{k=1}^{N}\left[T\left(\hat{\varepsilon}_{r}(\omega), h\right)-T_{\mathrm{FTS}_{k}}\right]^{2}
$$

where $N$ is the number of data points, $T$ the modeled transmittance, $T_{\text {FTS }}$ the measured transmittance data, and $h$ the measured sample thickness. We are guided by the Kramers-Kronig relations in defining constraints for a passive material: $\left|\hat{\varepsilon}_{j}\right|>\left|\hat{\varepsilon}_{j+1}\right|, \varepsilon_{j}^{\prime \prime}>0$ and $\hat{\varepsilon}_{r}(0)=\hat{\varepsilon}_{1}$ [16]. For accurate parameter determination, the sample should have uniform thickness, be adequately transparent to achieve high signal to noise, and have diffuse scattering as a subdominate process. The method requires an a posteriori numerical verification for Kramers-Kronig consistency. In the example presented here, a numerical Hilbert transform [17] of $\varepsilon_{r}^{\prime}(\omega)$ reproduces $\varepsilon_{r}^{\prime \prime}(\omega)$ to within $2 \%$ (Fig. 3). An alternative method employing reflectivity and phase allows a priori Kramers-Kronig consistent results [18]. However, given the details of the thin-film samples and available instrumentation, this approach was not implemented.

Figure 1 illustrates the measured and modeled results obtained from the analysis of a $0.5 \mu \mathrm{m}$ thick sample. The peak residual in the transmittance is less than $3 \%$, and the $3 \sigma=0.023$ uncertainty band indicated corresponds to the $99.7 \%$ confidence level. The standard deviation adopted for the measured data, $\sigma$, was estimated assuming the errors as a function of frequency were uniform and had a reduced $\chi^{2}$ equal to unity. An additional

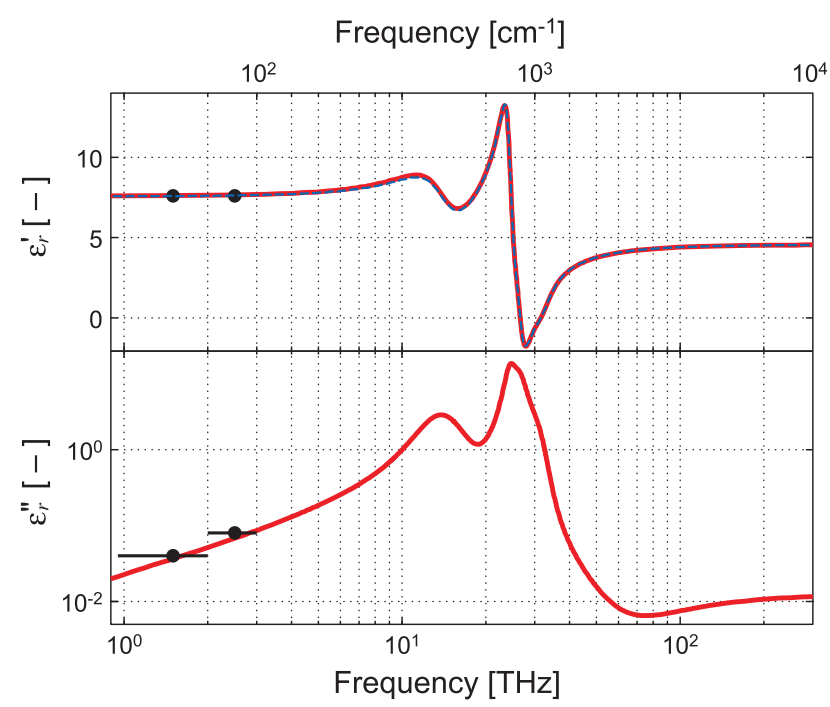

Fig. 3. (Color online) Real and imaginary parts (solid red curves) of the dielectric function as extracted from the data shown in Fig. 1. The line thickness is indicative of the propagated $\sim 4 \%$ error band. The numerical Hilbert transform of the modeled $\varepsilon_{r}^{\prime \prime}(\omega)$ is indicated in the upper panel (dashed blue line) to facilitate comparison with $\varepsilon_{r}^{\prime}(\omega)$. The filled symbols indicate the parameters derived from the data presented in Fig. 2 . 
Table 1. Fit Parameter Summary

\begin{tabular}{lllccc}
\hline$j[-]$ & $\varepsilon_{j}^{\prime}[-]$ & $\varepsilon_{j}^{\prime \prime}[-]$ & $\omega_{T_{j}} / 2 \pi[\mathrm{THz}]$ & $\Gamma_{j} / 2 \pi[\mathrm{THz}]$ & $\alpha_{j}[-]$ \\
\hline 1 & 7.582 & 0 & 13.913 & 5.810 & 0.436 \\
2 & 6.754 & 0.3759 & 15.053 & 2.751 & 0.0001 \\
3 & 6.601 & 0.0041 & 24.521 & 3.482 & 0.3427 \\
4 & 5.430 & 0.1179 & 26.440 & 5.948 & 0.0006 \\
5 & 4.601 & 0.2073 & 31.724 & 0.0080 \\
6 & 4.562 & 0.0124 & & \\
\hline
\end{tabular}

uncertainty in the FTS normalization influences the dielectric response function at the $1 \%$ level. In addition to the channel spectra, the observed spectrum shows two predominant features at 12 and 25 THz. Simulations with $M=2$ oscillators lead to a peak residual on transmission of $5 \%$ and do not enable recovery of the resonance at $25 \mathrm{THz}$. Using five oscillators satisfactorily recovers the observed transmittance and reduces the peak residual by a factor of 4.4. When the resonator's quality factor, $Q_{\operatorname{eff}_{j}}=\omega_{T_{j}} / \Gamma_{j}^{\prime}$, is greater than 5 , the data were not reproducible by either a pure Lorentzian oscillator or Eq. (4.6) in [12]. In these regions, the peak transmission residuals were decreased by a factor $\sim 2$ through the use of Eq. (2).

In Fig. 3 the values of the real and imaginary components of the dielectric function are illustrated as a function of frequency. The uncertainty in $\hat{\varepsilon}_{r}$ was propagated and computed as described in [19]. Table 1 contains a summary of the best fit parameters for five oscillators, which can be used to reproduce the data shown in Fig. 3.

To characterize the long-wavelength portion of the dielectric function, Fabry-Perot resonators were realized from one-, two-, and three-layer samples. Representative data for the three-layer resonator stack are presented in Fig. 2. A multilayer transfer matrix analysis [13] is used to extract the dielectric function using the measured $\mathrm{SiN}_{x}$ $(2.3 \mu \mathrm{m})$ and silicon spacer $(998 \mu \mathrm{m})$ thicknesses. The circular symbols at 1.5 and $2.5 \mathrm{THz}$ indicated in Fig. 3 were computed from a composite analysis of the three FabryPerot measurement sets. The horizontal range indicates the data used in each fit. The best estimates are $\hat{\varepsilon}_{r} \approx 7.6+$ $i 0.08$ over the range of $2-3 \mathrm{THz}$ and $\hat{\varepsilon}_{r} \approx 7.6+i 0.04$ over $0.4-2 \mathrm{THz}$. The real component of the static dielectric function derived from the data is in agreement with prior reported parameters for this stoichiometry [4]. As shown in Fig. 3, the measurements are internally consistent and represent roughly a factor-of-three reduction in uncertainty relative to prior IR $\mathrm{SiN}_{x}$ measurements identified by the authors [5-7]. The dielectric parameters reported here are representative of low-stress $\mathrm{SiN}_{x}$ membranes encountered in our fabrication and test efforts.

\section{References and notes}

1. D. J. Goldie, A. V. Velichko, D. M. Glowacka, and S. Withington, Appl. Phys. 109, 084507 (2011).

2. G. Wang, V. Yefremenko, V. Novosad, A. Datesman, J. Pearson, R. Divan, C. L. Chang, L. Bleem, A. T. Crites, J. Mehl, T. Natoli, J. McMahon, J. Sayre, J. Ruhl, S. S. Meyer, and J. E. Carlstrom, IEEE Trans. Appl. Supercond. 21, 232 (2011).

3. J. M. Martinis, K. B. Cooper, R. McDermott, M. Steffen, M. Ansmann, K. D. Osborn, K. Cicak, S. Oh, D. P. Pappas, R. W. Simmonds, and C. C. Yu, Phys. Rev. Lett. 95, 210503 (2005).

4. H. Paik and K. D. Osborn, Appl. Phys. Lett. 96, 072505 (2010).

5. T. Eriksson, S. Jiang, and C. Granqvist, Appl. Opt. 24, 745 (1985).

6. E. A. Taft, J. Electrochem. Soc. 118, 1341 (1971).

7. E. D. Palik, Handbook of Optical Constants of Solids (Elsevier, 1998), Vol. 1, pp. 771-774.

8. M. Sekimoto, H. Yoshihara, and T. Ohkubo, J. Vac. Sci. Technol. 21, 1017 (1982).

9. T. Makino, J. Electrochem. Soc. 130, 450 (1983).

10. Addison Engineering, 150 Nortech Parkway, San Jose, California 95134 (Orientation $\langle 100\rangle$, Czochralski, p-type B doped, bulk resistivity $<0.005 \Omega \mathrm{cm}$ ).

11. F. Gervais, in Infrared and Millimeter Waves, K. J. Button, ed. (Academic, 1983), Vol. 8, Part I, pp. 284-287.

12. C. C. Kim, J. W. Garland, H. Abad, and P. M. Raccah, Phys. Rev. B 45, 11749 (1992).

13. P. Yeh, Optical Waves in Layered Media (Wiley, 1988), pp. 102-111.

14. M. C. Biggs, in Towards Global Optimization, L. C. W. Dixon and G. P. Szergo, eds. (North-Holland, 1975), pp. 341-349.

15. M. J. D. Powell, in Mathematical Programming: The State of the Art, A. Bachem, M. Grotschel, and B. Korte, eds. (Springer Verlag, 1983), pp. 288-311.

16. L. D. Landau and E. M. Lifshitz, Electrodyamics of Continuous Media (Pergamon, 1960), Vol. 8, pp. 253-262.

17. M. Mori and T. Ooura, in Applicable Analysis (World Scientific Series, 1993), Vol. 2, pp. 301-308.

18. R. Nitsche and T. Fritz, Phys. Rev. B 70, 195432 (2004).

19. W. H. Press, S. A. Teukolsky, W. T. Vetterling, and B. P. Flannery, Numerical Recipes-The Art of Scientific Computing (Cambridge Univ., 2007), pp. 799-806. 